\title{
Síndrome del canal radial: nueva técnica de liberación endoscópica
}

\author{
J. Palau Gonzalez \\ Centre de la Mà de Barcelona. España.
}

\begin{abstract}
Resumen: El objetivo de este trabajo es presentar una nueva técnica endoscópica para la liberación de la rama motora del nervio radial en el síndrome del canal radial.

El síndrome del canal radial es la neuropatía compresiva de la rama motora del nervio radial que, muy a menudo, requiere la liberación quirúrgica mediante la apertura completa de las estructuras que producen la compresión.

Las vías de abordaje convencionales son amplias, requieren disecciones agresivas y laboriosas. El pequeño tamaño del nervio y su posición profunda entre vientres musculares hace que, fácilmente, pueda ser dañado durante su disección si la visualización no es correcta en todo momento.

Presentamos una nueva técnica que combina el marcaje preoperatorio de la posición del nervio mediante ecografía y liberación mediante técnica endoscópica. Esta técnica permite un abordaje quirúrgico mucho menor, una mejor visualización del nervio en todo su recorrido y de las estructuras anatómicas durante la disección.

Se presenta una serie de 22 intervenciones en 20 pacientes intervenidos entre 2010 y 2014. En 14 casos los resultados fueron excelentes, 5 buenos y 3 regulares. Las complicaciones fueron en un caso una lesión parcial del nervio durante la cirugía y 4 casos de paresia a la extensión de los dedos que se recuperaron completamente en todos los casos entre las 3 y 6 semanas.
\end{abstract}

Palabras claves: canal radial, interóseo posterior, endoscopia, ecografía

\section{Radial tunnel syndrome: new endoscopic technique release}

\begin{abstract}
The purpose is to present a new endoscopic technique for radial tunnel syndrome release.
The radial tunnel syndrome is the compressive neuropathy of the motor branch of the radial nerve that very often needs for surgical release by opening the anatomic structures that produces the compression.

The classical surgical approaches are large and aggressive. On the other hand the small size of the nerve and its deep position among several muscles are an important risk factor for damaging it during the surgery if the visualization of the nerve is not perfect during all the process.

The technique that we present combines the ultrasonographic identification of the nerve and skin drawing of the position and direction of it and endoscopic release. This method permits a smaller surgical approach and better visualization of the nerve during all the surgery.

We've done 22 interventions in 20 patients between 2010 and 2014. 14 has excellent results, 5 good and 3 fair. The complication was I partial nerve lesion during the surgery and 4 cases of temporary paresia with complete recovery after 3-6 weeks in all cases.
\end{abstract}

Key words: Radial tunnel, posterior interosseous nerve, endoscopy, ultrasonography.

El síndrome del canal radial (SCR) o neuropatía por atrapamiento del nervio interóseo posterior (NIP) sigue siendo hoy en día un diagnóstico controvertido tanto en el procedimiento diagnóstico como en su tratamiento.

A pesar de que el SCR fue descrito en 1972 por Roles y Maudsley', aún hoy en día su diagnóstico sigue siendo difícil. Los síntomas que refiere el paciente suelen ser confusos, las maniobras de provocación son difíciles y a menudo pueden desencadenar dolor producido por otras patologías de localización similar.
Además, casi en un $60 \%$ de los casos, se presenta en el contexto de las llamadas "epicondilitis resistentes" en que se combinan diferentes patologías simultáneamente, lo que dificulta aún más su diagnóstico.
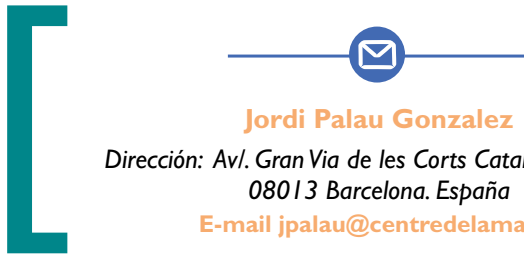

Dirección: Avl. Gran Via de les Corts Catalanes, 7I 7, 7. 08013 Barcelona. España

E-mail jpalau@centredelama.co 
También el hecho de que se trate de un nervio motor, como en el caso del nervio interóseo anterior, produce que el cuadro clínico se presente en forma de dolor difícil de explicar para el paciente y de localización e intensidad cambiante y sin clínica sensitiva. La electromiografía (EMG) presenta una muy baja sensibilidad y especificidad para esta patología, por lo que su utilidad es escasa y el pequeño tamaño del nervio hace que los cambios patológicos que presenta sean muy difíciles de detectar mediante prueba de imagen como la RM².

Sin embargo, la ecografía permite una visualización detallada tanto del nervio en todo su recorrido como de las estructuras anatómicas adyacentes que pueden participar de su compresión ${ }^{3}$. Por ello, para nosotros es una herramienta clave para el diagnóstico de esta neuropatía compresiva.

En cuanto a los abordajes quirúrgicos clásicos:

Clásicamente se han propuesto dos vías de abordaje: la anterior y la postero-lateral lateral o vía de Kaplan.

Abordaje anterior: Se aborda el espacio entre el braquiorradialis y el tendón del bíceps. La disección es compleja por las estrechas relaciones anatómicas de estructuras neurovasculares en la zona pero proporciona una buena visión de la arcada de Frohse. Sin embargo resulta muy difícil realizar una apertura completa del fascículo superficial del supinador hasta la arcada distal por lo que no se consigue liberar las compresiones a este nivel. En nuestra opinión este abordaje no es aconsejable en aquellos pacientes que puedan presentar una compresión a nivel de la arcada distal.

Abordaje lateral por el intervalo de Kaplan: Aborda el supinador a través del intersticio entre el extensor carpi radialis brevis (ECRB) y el extensor digitorum comunis (EDC). La apertura completa del supinador obliga a realizar un abordaje muy largo que permita una amplia exposición del supinador y la incisión completa de la fascia profunda del ECRB. Ello implica una importante agresión quirúrgica de la zona debiendo disecar a dos niveles diferentes si se quiere liberar el canal en toda su extensión. Además la visualización de la arcada de Frohse por esta vía es dificultosa con lo cual la apertura de las fibras proximales se realiza casi a ciegas con el consiguiente riesgo de lesión del nervio.
La introducción de técnicas endoscópicas nos ha permitido realizar abordajes quirúrgicos mucho más pequeños y mejorar al mismo tiempo la visualización de las estructuras nerviosas, amplificándolas, lo cual permite un mejor control de la disección. El estudio y marcaje ecográfico del nervio previo a la liberación endoscópica permite una mejor comprensión y planificación preoperatoria que es esencial en las técnicas endoscópicas donde la incisión cutánea es mínima.

El objetivo del presente trabajo es la descripción de una nueva técnica de liberación endoscópica del nervio Interóseo posterior y la descripción del estudio ecográfico previo que facilita su realización.

\section{INDICACIONES}

\section{Criterios diagnósticos}

Expuesto lo anterior proponemos los siguientes criterios diagnósticos:

\section{Criterios mayores:}

I) Clínica y exploración física compatibles. Historia clínica de dolor de más de 3 meses de evolución.

2) Hallazgos ecográficos patológicos en el aspecto del nervio compatibles con neuropatía de atrapamiento (engrosamiento hipoecoico y pérdida del patrón fascicular) en zonas anatómicas susceptibles de ser compresivas.

3) Presencia de otras patologías locales que puedan afectar al nervio como sinovitis de la articulación radio-humeral, gangliones sinoviales, tumoraciones, exostosis $u$ otras lesiones osteoarticulares de la articulación radiohumeral.

\section{Criterios menores:}

I) Sintomatología en territorios distales como dorso antebrazo, muñeca y dorso de la primera comisura dela mano.

2) Ausencia de hallazgos patológicos en el estudio ecográfico pero dolor a la compresión local del nervio a nivel de arcada proximal y distal del canal radial. 

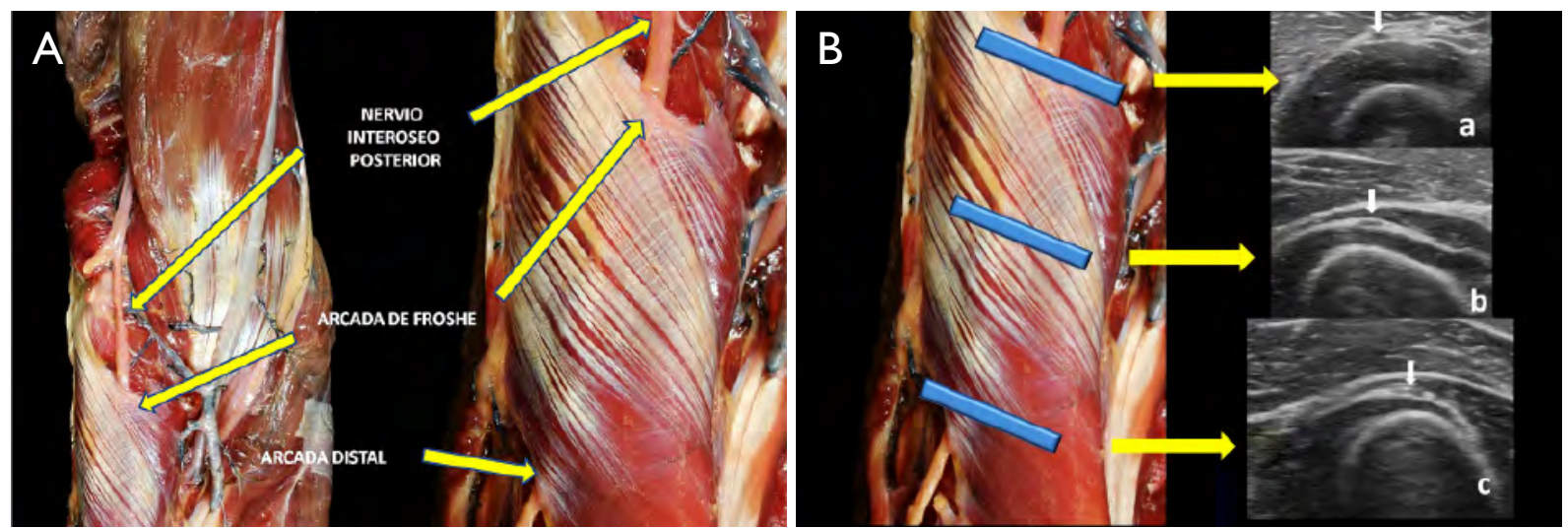

Figura I. Anatomía y exploración ecográfica del canal radial. A. Disección anatómica del nervio radial en la región anterior del codo. Se aprecia la división del nervio radial y el detalle anatómico de la rama motora introduciéndose bajo la arcada de Frohse y cómo emerge bajo la arcada distal. B. Imagen ecográfica correspondiente a los tres niveles del canal radial. Se indica con flechas blancas la posición de la rama motora en cada nivel. a) Bajo la arcada de Frohse. b) En el tercio medio del espacio entre los fascículos superficial y profunda del supinador. c) A la salida distal bajo la arcada distal del supinador.

\section{Indicaciones del tratamiento quirúrgico}

Nosotros indicamos tratamiento quirúrgico a aquellos pacientes que cumplen 2 de los 3 criterios mayores y a aquellos pacientes que cumpliendo el criterio mayor I y uno o varios de los criterios menores no han respondido al tratamiento conservador.

\section{ANATOMÍA QUIRÚRGICA}

\section{Exploración ecográfica prequirúrgica}

De cara a la planificación quirúrgica procedemos a una exploración física y ecográfica del nervio en todo su recorrido.

\section{Consideraciones anatómicas:}

Entendemos como canal radial la región anatómica por donde discurre la rama motora del nervio radial, desde su bifurcación de la rama sensitiva en la fosa bicipital externa del codo aproximadamente en la zona anterior al capitellum humeral hasta su salida distal entre los fascículos superficial y profundo del supinador, donde pasa a denominarse nervio interóseo posterior y se divide en sus ramos terminales.

En este recorrido el nervio se relaciona anatómicamente con la fascia profunda del ECRB, bandas fibrosas provenientes de fascias musculares vecinas, estructuras vasculares correspondientes a la arteria comunicante radial, hasta introducirse entre los fascículos superficial y profundo del supinador. A la entrada de este espacio cruza por debajo de una banda fibrosa que de hecho se corresponde con la porción libre y proximal de la fascia del fascículo superficial del supinador y que conocemos como Arcada de Frohse.

Una vez entre los dos fascículos del supinador, el nervio sigue un recorrido oblicuo rodeando el cuello del radio hasta emerger bajo el límite inferior del fascículo superficial en la zona dorsal del antebrazo, para ir a buscar la superficie dorsal de la membrana interósea y donde recibirá el nombre de nervio interóseo posterior (NIP).

A la salida distal del fascículo superficial del supinador hay también una fascia fibrosa casi constante y que puede ser un punto de compresión igual que lo es la arcada de Frohse ${ }^{4,5}$.

Clásicamente se ha identificado a la arcada de Froshe y las estructuras anatómicas de la región proximal del canal radial como las únicas estructuras responsables de la compresión del NIP. Sin embargo, hemos observado que pueden producirse compresiones del nervio a nivel de la arcada distal de forma aislada hasta en un 30\% de los casos y en un 10\% combinada con compresiones en la arcada de Frohse, es decir, compresiones dobles.

Por este motivo, en un porcentaje de casos importante, se requiere una liberación quirúrgica tanto de la zona proximal como de la distal.

\footnotetext{
Sistemática y objetivos de la exploración ecográfica.

Durante la exploración ecográfica se puede localizar el NIP en todo su recorrido y en los puntos de posible compresión se puede evaluar su morfología.
} 


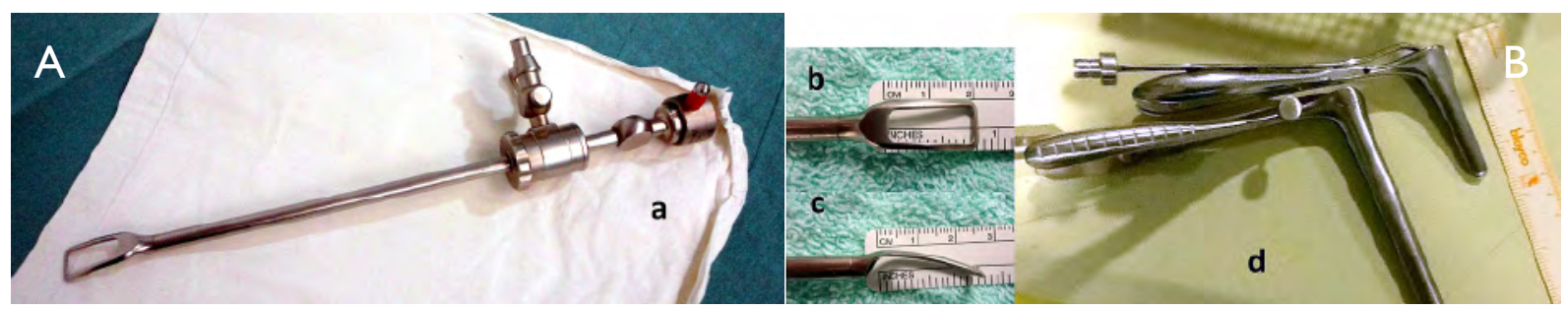

Figura 2. Material específico para la técnica endoscópica. A. Óptica con espéculo separador de partes blandas adaptado en su extremo (Endoscope Storz ${ }^{\circledR}$ ). B. Detalle del espéculo sobre regla milimetrada. Especulo intranasal modificado al que se le ha adaptado un tornillo para su fijación en una posición estática y adaptador para fuente de luz que facilita la visión durante su introducción.

Se deben localizar sistemáticamente las siguientes estructuras (2) Figura I.

- Tronco común del nervio radial y su bifurcación.

- Arteria comunicante radial y arcada de Frohse.

- Recorrido del nervio motor entre los fascículos del supinador.

- Arcada distal del supinador y división de las ramas terminales recurrente y profunda.

Al igual, se deben buscar cambios en la ecoestructura del NIP en los posibles puntos de posible compresión. En caso de una neuropatía compresiva, los nervios presentan una dilatación brusca de su tamaño, con pérdida del patrón fascicular normal por el edema intraneural. Es decir, que no se puede diferenciar en su interior los diferentes fascículos nerviosos y este segmento de nervio se vuelve más oscuro (hipoecoico). Todo ello corresponde con los cambios propios de un neuroma por compresión.

Hay que destacar que una neuropatía compresiva puede mostrar un nervio de aspecto ecográfico totalmente normal durante un período de meses o años desde el inicio de los síntomas. Hemos denominado a este periodo de tiempo como "estadio pre-ecográfico".

Para evaluar clínicamente, durante este estadio preecográfico, si el nervio está comprimido y si es la causa del cuadro doloroso, procedemos a localizar el nervio justo en el punto de posible compresión y, bajo control ecográfico, comprimimos el nervio con el dedo índice de la mano contralateral a la que usamos el transductor con una presión aproximada de I kg. Si esta maniobra reproduce el dolor motivo de consulta y es clínicamente compatible con el diagnóstico, consideramos que es altamente probable de que se trate de una neuropatía por compresión del NIP.
Esta maniobra compresiva se debe realizar de forma sistemática a cuatro niveles diferentes: sobre la arcada de Froshe, sobre la arcada de salida distal del canal, aproximadamente a medio recorrido del canal radial y una cuarta compresión sobre el vientre muscular del braquiorradialis, fuera del recorrido del canal como "punto de control". Este punto no debería ser doloroso en ningún caso, por lo que si el paciente se queja y/o el resto de la exploración es poco congruente, debemos concluir que se puede tratar de otra patología.

Hemos realizado estas maniobras en 20 voluntarios sanos y en ningún caso ha resultado dolorosa.

\section{TÉCNICA QUIRÚRGICA}

La introducción de técnicas endoscópicas nos ha permitido realizar abordajes quirúrgicos mucho más pequeños y mejorar al mismo tiempo la visualización de las estructuras.

\section{Instrumental}

Para la realización de esta técnica endoscópica se utiliza el instrumental introducido y diseñado por Reimer Hoffman para la liberación del nervio cubital ${ }^{6}$, junto con el instrumental necesario para la exploración ecográfica.

El material básicamente consta de dos componentes (1) Figura 2.

Un espéculo intranasal modificado con un tornillo, que permite mantener una apertura constante, a modo de separador autoestático y un adaptador para la fuente de luz.

Una cánula de artroscopia de 4,2 mm, como la usada en artroscopia de rodilla, a la que se le ha adaptado una paleta en el extremo distal a modo de separador de partes blandas. 

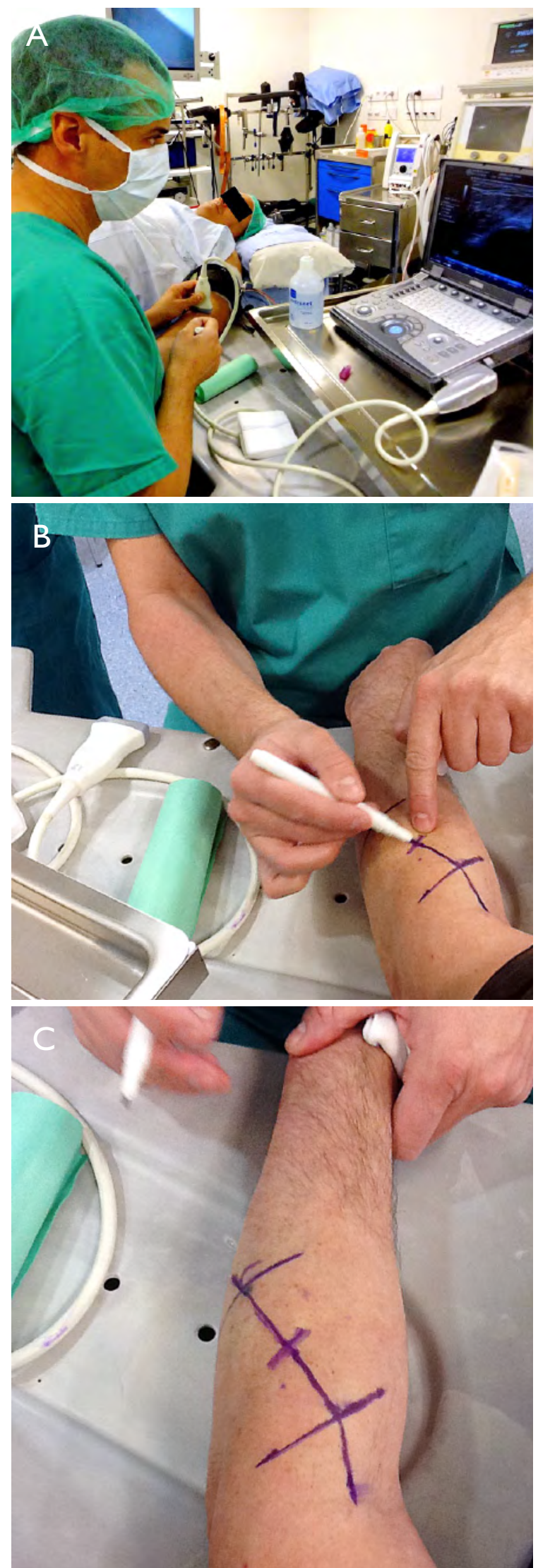

Figura 3. Imágenes del proceso de marcado cutáneo. A. Posición del paciente en la mesa quirúrgica y disposición del ecógrafo que debe situarse en el lado del hombro del paciente. Es importante realizar el marcaje con la extremidad en la misma posición en la que va a ser intervenida. B. Proceso de marcaje con un rotulador demográfico. C. Marcaje final en el que se aprecia una línea longitudinal oblicua que indica la posición y dirección del nervio y tres líneas transversales que de proximal a distal indican la posición de la Arcada de Frohse, el intervalo ECRB-EDC y la arcada distal.

\section{Colocación del paciente}

Con el paciente en decúbito supino, la extremidad apoyada sobre la mesa de mano y con el manguito de isquemia preparado en la extremidad. Es preferible usar una técnica de bloqueo anestésica del plexo que nos permita usar un manguito de isquemia simple y situado lo más proximal posible en el brazo. El cirujano deberá situarse en la parte del hombro del paciente mientras que el ayudante deberá situarse a la axila del paciente.

\section{Marcaje cutáneo del trayecto del nervio y limites anatómicos}

El primer paso consiste en la localización del nervio motor con el ecógrafo en todo su recorrido, desde la bifurcación del nervio radial a nivel de la fosa anterradial del codo hasta su salida distal del canal radial.

Con un rotulador dermográfico se dibuja sobre la piel todo el recorrido del nervio. Para ello se marcan diversos puntos sobre el recorrido del nervio y se unen por una línea. Esta marca indica la posición y dirección del nervio y permitirá en todo momento saber si la orientación del instrumental es correcta. Es muy importante dedicar unos minutos al marcaje cutáneo correcto y realizarlo con el antebrazo en pronación y en la misma posición en que se va a realizar la cirugía ya que un cambio de posición de la extremidad comportará un cambio de posición del nervio en relación al marcaje cutáneo, lo que podrá derivar en la dificultad de localización o en la lesión del nervio durante la disección.

A continuación se marcan las arcadas proximal (Fröhse) y distal sobre la piel para establecer los límites del canal radial @ Figura 3.

Por último, se localiza el intervalo entre ECRB y el EDC (intervalo de Kaplan) y de nuevo se dibuja sobre la piel. Aunque es posible localizar dicho espacio simplemente por palpación a menudo puede ser difícil en pacientes obesos o con vientres musculares poco definidos. 

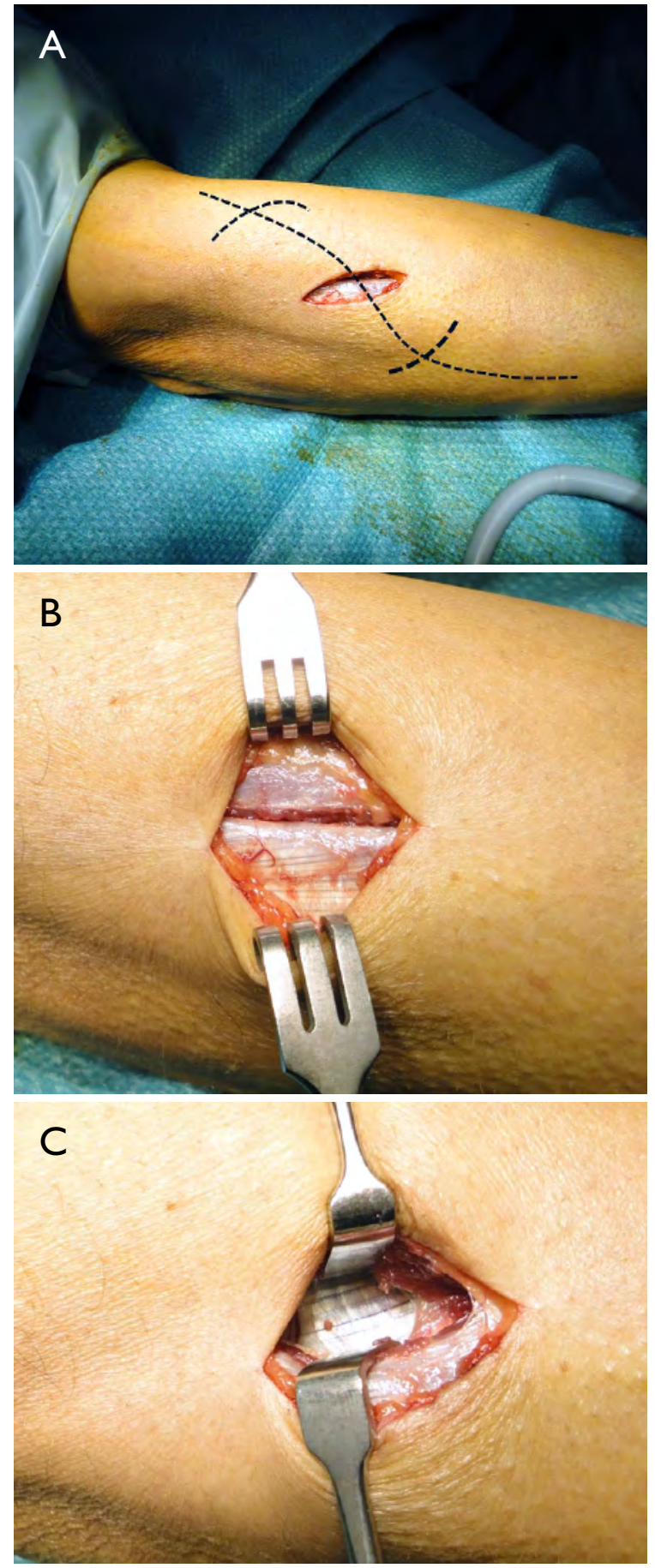

Figura 4. Vía de abordaje. A. Incisión cutánea de unos 3-4 cm siguiendo el intervalo ECRB-EDC o via de Kaplan. Nótese que este intervalo está más próximo a la arcada distal que a la propia arcada de Frohse. B. Apertura de la fascia e identificación del espacio entre los vientres musculares. C. En la porción profunda del intervalo y tras abrir parcialmente la fascia profunda del ECRB aparece la fascia superficial del supinador con su característico aspecto atigrado y con las fibras en dirección claramente oblicua respecto a la dirección de los vientres musculares de ECRB y EDC.
Un detalle importante es utilizar poca cantidad de gel conductor y evitar el contacto del rotulador con el gel puesto que queda inmediatamente inservible. Para ello es práctico realizar inicialmente unas marcas con el rotulador, limpiar completamente el gel de la piel y finalmente unir las líneas con el rotulador con la piel ya libre de gel.

\section{Técnica quirúrrgica endoscópica}

Tras el estudio ecográfico y el marcaje cutáneo, se realiza la isquemia de la extremidad.

La incisión cutánea es aproximadamente de $3-4 \mathrm{~cm}$ sobre el intervalo ECRB-EDC. Se diseca el tejido subcutáneo con atención de no lesionar la rama cutánea antebraquial dorsal y se practica una incisión de la fascia antebraquial siguiendo la dirección del intervalo muscular.

Se identifica el plano muscular entre ECRB y EDC que se diseca fácilmente de forma roma hasta identificar la fascia superficial del fascículo superficial del supinador. Esta fascia presenta un aspecto muy característico ya que sus fibras discurren de forma completamente oblicua al sentido del resto de la musculatura extensora del antebrazo y su aspecto es atigrado (1) Figura 4.

En este punto introducimos el espéculo en sentido proximal sobre la superficie del supinador, se sigue la dirección del nervio siguiendo la línea que previamente se ha dibujado sobre la piel e introducimos la óptica en la dirección indicada (aigura 5.

El primer paso consiste en seccionar la fascia profunda del ECRB. Esta es una fascia gruesa y tensa y que se ha descrito como uno de los elementos que puede contribuir a la compresión. La sección de dicha fascia se realiza en el mismo sentido del espéculo, realizando la sección entre las dos valvas. Se localiza por encima del instrumental a modo de "techo" del túnel que se ha disecado. Esta maniobra facilita el poderse mover con el instrumental en un espacio de por sí muy reducido. Siguiendo estas indicaciones, nos encontraremos aproximadamente a la mitad del canal radial.

A continuación se realiza una disección roma del fascículo superficial del supinador hasta identificar la rama motora del radial (RMR) que, si se han realizado correctamente los marcajes cutáneos, debería aparecer en el mismo plano de la disección. Este paso es crítico en esta técnica, puesto que realizamos una búsqueda del nervio a través del músculo a ciegas, por 


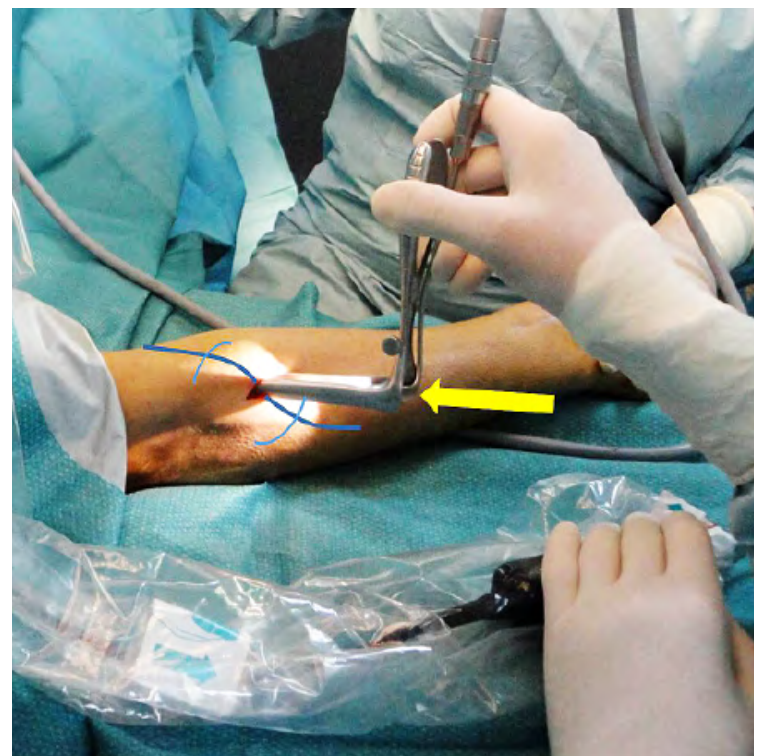

Figura. 5. Introducción del espéculo y la óptica en sentido proximal y siguiendo la dirección de la marca cutánea que indica la posición y dirección del nervio.

lo que debemos extremar las precauciones para no lesionar el nervio antes de identificarlo.

Una vez identificada la RMR proseguimos con la disección del fascículo del supinador en dirección proximal hasta la arcada de Fröhse, que seccionaremos. Procedemos a la disección en sentido proximal de la RMR hasta identificar los ramos vasculares que la cruzan (arteria comunicante radial o Leash of Henry). Si no se aprecian evidencias de compresión del nervio a este nivel se conservarán dichos vasos y se concluirá la disección en sentido proximal, retirando con cuidado el instrumental a la vez que se comprueba visualmente que el nervio está completamente liberado (1) Figura 6.

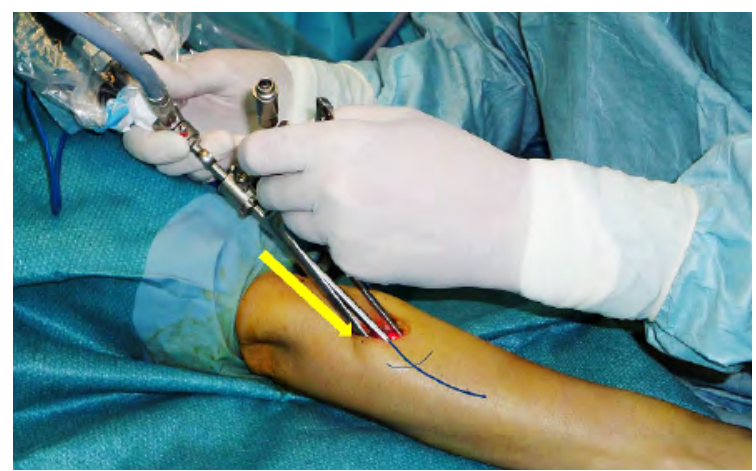

Figura 7. Introducción del espéculo y la óptica esta vez en sentido distal y nuevamente siguiendo como referencia el marcaje cutáneo.

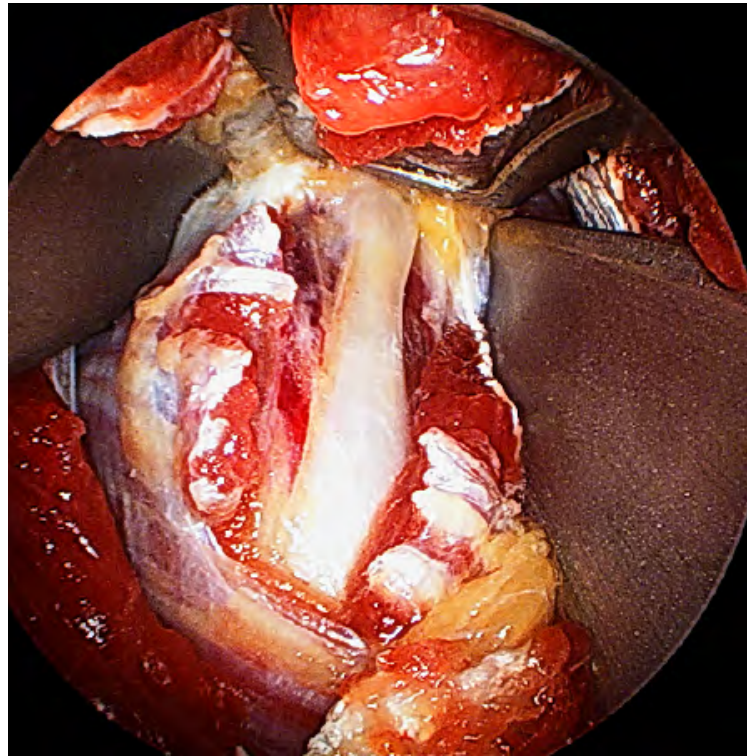

Figura 6. Imagen de la rama motora liberada a nivel de la arcada de Frohse. Nótese la imagen en reloj de arena debida a la compresión.

Se retira el instrumental y se da por finalizada la liberación proximal.

A continuación se procede con la liberación en sentido distal. Ésta es bastante más difícil que la anterior, puesto que la posición será muy incómoda y debe ser realizada con máximo cuidado.

Para ello se introduce de nuevo el separador, esta vez en sentido distal y siguiendo la posición y dirección del nervio que hemos dibujado en la piel (a) Figura 7.

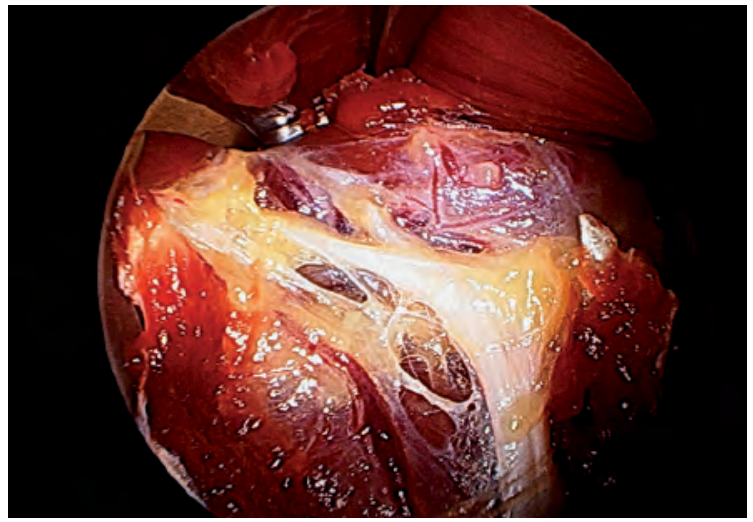

Figura 8. Imagen de la liberación a nivel de la arcada distal. Se aprecia claramente una dilatación del nervio distal al punto de liberación. En este punto la rama motora del nervio radial pasa a denominarse nervio interóseo posterior. Es importante identificar en todo momento las ramas distales para no lesionarlas durante la disección. 
Tomando como plano el supinador e identificando de nuevo la RMR en la zona más distal de la disección anterior, introducimos el separador siguiendo la dirección de la marca cutánea en sentido distal. Se introduce la óptica y se prosigue con la disección en sentido distal del nervio hasta identificar el margen distal del fascículo superficial del supinador, que habitualmente aparece como una banda fibrosa fácil de identificar y que deberá seccionarse completamente.

Es muy importante visualizar correctamente el nervio durante toda su disección y especialmente en la arcada distal ya que justo a su salida el NIP emite una rama motora recurrente que retrocede sobre la superficie distal del supinador para inervar el EDC y que fácilmente puede lesionarse durante la disección.

Liberada la arcada distal se identifica cómo el nervio se bifurca y no es necesario proseguir más distalmente con lo que se puede retirar el instrumental y dar por finalizada la liberación del NIP @ Figura 8.

Se sutura la fascia superficial del intervalo EDC-ECRB, tejido subcutáneo y la piel. Generalmente se realiza una sutura intradérmica para mejorar el resultado estético final de la cicatriz.

\section{MANEJO POSTOPERATORIO}

Se realiza un vendaje almohadillado del codo sin limitar la movilidad del mismo.

A los 5-7 días se retira el vendaje almohadillado, dejando un simple apósito que el paciente puede cambiar a diario y se retira la sutura a las dos semanas de la cirugía.

Habitualmente, como en la mayoría de cirugías de neuropatías compresivas, no se requiere de rehabilitación posterior. Sin embargo, en pacientes con síntomas de larga evolución suelen asociarse molestias y alteraciones miofasciales de la zona que deben ser tratadas.

\section{SERIE DEL AUTOR}

Entre 2010 y 2014 se ha realizado un total de 22 intervenciones mediante la técnica endoscópica en 20 pacientes.

La distribución por sexos fue de 13 mujeres y 7 hombres con una media de edad de 41,25 años (rango
24-5 I años). En I 5 pacientes la extremidad afecta fue la dominante, en 3 la no dominante y en 2 bilateral.

Hay que destacar el largo periodo transcurrido entre la aparición de los síntomas y el tratamiento. El promedio de tiempo fue de 28 meses (rango de 2 a 84 meses) destacando que en 10 de los pacientes el diagnóstico se retardó más de 24 meses.

16 de los 20 pacientes desarrollaban profesiones consideradas de esfuerzo manual repetitivo, mientras que 4 desarrollaban profesiones liberales (ingeniería, docencia) pero todos ellos coincidían con el uso intensivo de ordenadores personales.

15 de los 20 pacientes presentaban otras patologías asociadas como epicondilitis, síndromes del meniscoide radio-humeral, otras neuropatías compresivas del codo y entesopatías del tríceps. 3 de ellos presentaban simultáneamente una neuropatía de atrapamiento de la rama sensitiva del radial o síndrome de Wartemberg de la misma extremidad. De estos 15 , II presentaban simultáneamente más de una patología asociada.

De ello se desprende que sólo el 25\% de los pacientes presentaban un síndrome del canal radial aislado, mientras que en el otro $75 \%$ de los pacientes este síndrome se encuentra inmerso y por tanto "camuflado" en el contexto de otros cuadros dolorosos del codo.

El periodo de tiempo transcurrido desde la cirugía hasta la desaparición del dolor atribuible a la compresión del nervio fue de 4 semanas (rango 1-32). Dos pacientes con clínica de más de un año de evolución antes de la cirugía presentaron persistencia de dolor hasta los 6 meses del postoperatorio. Sin embargo, no se ha observado que los pacientes con más tiempo de evolución clínica antes de la cirugía hayan presentado de forma significativa un periodo mayor de recuperación. Dos pacientes en que los síntomas habían aparecido hasta 6 años antes de la cirugía, presentaron mejoría clínica completa en unas pocas semanas.

Todos los pacientes de esta serie se reincorporaron a su puesto de trabajo habitual.

Dada la combinación en la mayoría de los casos de diferentes patologías ha sido difícil encontrar una escala de evaluación que nos permitiera categorizar los resultados en base únicamente a los síntomas del canal radial. Por ello se han considerado los resultados de la siguiente forma: 
Excelente: Desaparición completa de los síntomas atribuibles al síndrome del canal radial en un periodo inferior a las 4 semanas.

Bueno: Mejoría importante de las molestias en un periodo de 4 a 8 semanas del postoperatorio con una mejoría completa dentro de los 3 primeros meses.

Regular: Persistencia de síntomas aunque fueran ocasionales durante un periodo de más de 3 meses.

Malo: Sin mejoría clínica o empeoramiento de los síntomas después de la cirugía a los 6 meses.

En base a este criterio el resultado de las 22 intervenciones podemos distribuirlos: 14 resultados excelentes $(63,6 \%), 5$ buenos $(22,7 \%)$ y 3 regulares (13,6\%). En ningún caso el resultado ha sido malo, por ello deberíamos considerar que si la descompresión se ha realizado de forma correcta y los síntomas iniciales persistieran hay que plantearse que se pueda haber cometido un error diagnóstico como primera posibilidad.

En cuanto a las complicaciones observadas asociadas a la técnica:

En 4 intervenciones se presentó en el postoperatorio inmediato una paresia o debilidad para la extensión activa de los dedos. En todos los casos dicha paresia se recuperó completamente de forma espontánea en un periodo promedio de 4 semanas (rango 3-6 semanas). Estos casos los atribuimos inicialmente a la manipulación directa del nervio durante la cirugía. Sin embargo, hemos observado que durante el periodo postoperatorio casi la mitad de los nervios intervenidos han presentado un edema perineural importante en las semanas posteriores y muchos de ellos han desarrollado un halo hiperecoico perineural que podría interpretarse como un edema y posterior fibrosis post-descompresión. Estos cambios, difíciles de cuantificar, creemos que se debe a procesos de hiperemia post-descompresión que podrían ser normales y posiblemente causantes o corresponsables de las paresias que algunos pacientes han presentado. Dichos cambios en la ecoestructura del nervio pueden verse en algunos pacientes hasta 2 años después de la cirugía.

En un caso se produjo una sección parcial (aproximadamente 30\%) del nervio durante la disección. Dicha lesión se produjo por un error humano del cirujano que no advirtió que la orientación del instrumental había variado durante la disección respecto al mar- caje cutáneo por lo que el nervio apareció en sentido transversal en el campo quirúrgico y fue confundido con una estructura fascial. Se procedió a la sutura epineural con Ethilon 8/0. La paciente, que fue debidamente informada del incidente, presentó una paresia de extensión de $3^{\circ}-4^{\circ}$ y $5^{\circ}$ dedos que se recuperó completamente en un periodo de 10 semanas.

No se ha observado por el momento otras complicaciones como infecciones o síndrome de dolor regional complejo, que lógicamente podrían presentarse en esta técnica como en cualquier otra de estas características.

En cuanto a las ventajas que pensamos que esta técnica endoscópica aporta como ventajas respecto a la técnica abierta:

Menor tamaño de la incisión y menor agresión quirúrgica de la musculatura y estructuras fasciales, por lo que representa una mejoría tanto del tiempo de recuperación como una menor secuela estética.

Mejor visualización del nervio en todo momento mediante monitor y amplificación de la imagen, lo cual permite un mejor control de la disección.

El estudio y marcaje ecográfico del nervio o de cualquier estructura puede ser utilizado en cualquier técnica quirúrgica, pero su realización permite una mejor comprensión y planificación preoperatoria que es esencial en las técnicas endoscópicas, donde perdemos las referencias anatómicas que utilizamos en las técnicas abiertas.

La principal desventaja de esta técnica es que se requiere de una fundamentada formación en ecografía para poder realizar el diagnóstico y marcaje mediante esta técnica y que la técnica quirúrgica endoscópica es más exigente. Hoy en día la ecografía es una técnica en vías de expansión en nuestra especialidad pero seguro que en unos años pasará a ser una técnica habitual de exploración para el traumatólogo, igual que lo es para muchas otras especialidades. Asimismo, la habilidad y experiencia que hemos ido adquiriendo tanto en artroscopia de pequeñas articulaciones como en técnicas endoscópicas, nos hubieran parecido impensables hace unos años y son hoy en día una realidad. También pudo parecer impensable realizar una meniscectomía artroscópica hace décadas y hoy en día es una técnica absolutamente corriente y extendida. 


\section{AGRADECIMIENTOS:}

A Iván Sáenz, por la cesión de imágenes de disección anatómica.

\section{CONFLICTOS DE INTERESES}

Los autores declaran no tener conflictos de intereses.

\section{BIBLIOGRAFIA}

I. Roles NC, Maudsley RH. Radial tunnel syndrome. Resistant tennis elbow as a nerve Entrapment. J Bone J Surg Br. 1972;54(3):499-508.

2. Stanley J. Radial Tunnel Syndrome: A Surgeon's Perspective. J Hand Ther. 2006; 19(2): 180-4.
3. Carreras Burgaya A, Palau González J, Sala Blanch $X$. Ecografía del nervio periférico. En: Llusá Pérez (Ed). Anatomía quirúrgica del plexo braquial y de los nervios periféricos de la extremidad superior. Madrid: Médica Panamericana; 2013. p. 329-38.

4. Hazani R, Engineer NJ, Mowlavi A, Neumeister M, Lee WP, Wilhelmi BJ. Anatomic Landmarks for the radial tunnel. Eplasty. 2008;22:377-382.

5. Clavert P, Lutz JC, Adam P, Wolfram-Gabela R, Liverneauxb P, Kahna JL. Frohse's arcade is not the exclusive compression site of the radial nerve in its tunnel. Orthop Traumatol Surg Res. 2009;95: I I4118.

6. Hoffman R, Siemionov M. The endoscopic management of cubital tunnel syndrome. J Hand Surg Br. 2006;3I ( I):23-9. 